\title{
An imaging polarimeter (IMPOL) for multi-wavelength observations
}

\author{
A.N. Ramaprakash ${ }^{1}$, R. Gupta ${ }^{1}$, A.K. Sen $^{2}$, and S.N. Tandon ${ }^{1}$ \\ 1 Inter-University Centre for Astronomy and Astrophysics, Post Bag 4, Ganeshkhind, Pune - 411 007, India \\ 2 Department of Physics, Assam University, Silchar - 788 001, India
}

Received January 20; accepted July 11, 1997

\begin{abstract}
Taking advantage of the advances in array detector technology, an imaging polarimeter (IMPOL) has been constructed for measuring linear polarization in the wavelength band from $400-800 \mathrm{~nm}$. It makes use of a Wollaston prism as the analyzer to measure simultaneously the two orthogonal polarization components that define a Stoke's parameter. An achromatic half-wave plate is used to rotate the plane of polarization with respect to the axis of the analyzer so that the second Stoke's parameter also can be determined. With a field of view corresponding to about $30 \times 30 \mathrm{~mm}^{2}$ for a $\varnothing 1.2 \mathrm{~m}, \mathrm{f} / 13$ telescope, a sensitive, liquid- $\mathrm{N}_{2}$ cooled $\mathrm{CCD}$ camera as the detector and a built-in acquisition and guidance unit, the instrument can be used for studying stellar fields or extended objects with an angular resolution of $\sim 2^{\prime \prime}$. The instrumental polarization is less than $0.05 \%$ and the accuracies of measurement are primarily limited by photon noise for typical observations.
\end{abstract}

Key words: instrumentation: polarimeters - techniques: polarimetry — polarization

\section{Introduction}

The advances in two dimensional array detector technology in the optical and near infrared wavelength bands have made new kinds of imaging astronomical observations feasible. Astronomical polarimetry is one field which has gained tremendously from these developments. The limitations of using aperture photometry for polarimetry were so severe that any serious study was rendered time consuming and difficult. On the other hand, imaging polarimetry with its capabilities for multiplexing, simultaneous sky measurement, seeing-limited resolution etc. offer great advantages over aperture polarimetry. Astronomers have recognized this potential and have developed several

Send offprint requests to: A.N. Ramaprakash new observational techniques in the optical (e.g. Scarrott 1991; Jannuzi et al. 1993; Jarrett et al. 1994; Wolstencroft et al. 1995; Simmons et al. 1995) and near infrared (e.g. Kastner \& Weintraub 1994; Moore \& Yamashita 1995; Weintraub et al. 1995) wavelengths to study phenomena in a variety of Galactic and extragalactic astrophysical objects. In this paper, we report the design and construction of an imaging polarimeter (IMPOL) which uses a cooled CCD array as detector. It was developed at the Inter-University Centre for Astronomy and Astrophysics (IUCAA), India. The principle of the instrument (Sen \& Tandon 1994) is based on a combination of ideas suggested by Ohman (1939) and Appenzeller (1967). An instrument of this type has been constructed at the University of Durham and has been in use for some time now (Scarrott et al. 1983). Section 2 is a description of the instrument - the different subsections dealing with various aspects ranging from design guidelines to instrument control and user-interface. The dominant sources of errors in the measurement are investigated in Sect. 3, while an estimate of the performance of the instrument under two typical observing conditions is given in Sect. 4 . In Sect. 5 we discuss the observational procedure and the data-analysis method. Section 6 contains results obtained during commissioning of the instrument. The last section (Sect. 7) contains a summary.

\section{The instrument}

The following set of scientific and technical guidelines were adopted as basis for design of the instrument. (i) It should be capable of observing faint extended objects like reflection nebulae, accretion disks, dusty active galaxies etc., with accuracy limited by photon noise and resolution limited by seeing. This demands a typical field of view of a few arcminutes, an optics which is well-matched with the telescope to minimize loss of light and a detector of sufficient sensitivity. Also, the effects of atmospheric scintillation should be eliminated and instrumental polarization minimized so that such measurements of low level 
polarization could be made. (ii) Since the objects of interest are very often quite faint, the instrument should have an acquisition and guidance $(\mathrm{A} \& \mathrm{G})$ system which allows pointing the telescope with an accuracy of a few arcseconds and tracking to better than $1^{\prime \prime}$ over long periods. (iii) Observations should be possible in various wavelength bands in the optical and near-IR regions. (iv) The entire instrument consisting of the optics, A\&G unit, associated electronics etc. should be a self-contained unit which can be easily mounted on the telescope, with only electrically isolated communication links to the computers for instrument control and data acquisition. (v) The cost of the instrument should be minimized by using standard optical and electronic components and by avoiding overspecification as far as possible.

\subsection{Principle of the instrument}

Figure 1 is a schematic representation of the optical arrangement of IMPOL. The basic idea behind this arrangement is to use a Wollaston prism with its axis normal to the optical axis of the system, as the analyzer to convert the linear polarization in the incoming light into relative intensity of two orthogonally polarized beams (the ordinary and the extraordinary), separated by a small angle of $0.5^{\circ}$. This measurement is sufficient to define one of the Stoke's parameters $Q$ or $U$. A half-wave plate with its fast-axis normal to the optical axis of the system, is kept before the Wollaston prism on a rotatable mounting. When the half-wave plate is rotated through an angle $\alpha$, the plane of polarization rotates through an angle $2 \alpha$. At this new position of the half-wave plate another measurement on the orthogonally polarized beams can be made to determine the second Stoke's parameter as well. It is easily seen that, for this arrangement the intensities of the extraordinary and ordinary beams $\left(I_{\mathrm{e}}\right.$ and $\left.I_{\mathrm{o}}\right)$ are given by

$$
\begin{aligned}
& I_{\mathrm{e}}(\alpha)=\frac{I_{\mathrm{up}}}{2}+I_{\mathrm{p}} \cos ^{2}(\theta-2 \alpha) \\
& I_{\mathrm{o}}(\alpha)=\frac{I_{\mathrm{up}}}{2}+I_{\mathrm{p}} \sin ^{2}(\theta-2 \alpha),
\end{aligned}
$$

where $I_{\mathrm{up}} \& I_{\mathrm{p}}$ are the unpolarized and polarized intensities respectively; $\theta \& \alpha$ are the position angles of the polarization vector and the half-wave plate fast-axis respectively, with reference to the axis of the Wollaston prism. Since the angle $\theta$ is conventionally measured with respect to the celestial north-south axis ( 0 deg towards north celestial pole and increasing counter-clockwise), the axis of the Wollaston prism is kept aligned to it.

We define the ratio

$$
R(\alpha)=\frac{I_{\mathrm{e}} / I_{\mathrm{o}}-1}{I_{\mathrm{e}} / I_{\mathrm{o}}+1}=p \cos (2 \theta-4 \alpha),
$$

where $p=\frac{I_{\mathrm{p}}}{I_{\mathrm{up}}+I_{\mathrm{p}}}$, is the fraction of the total light in linearly polarized condition. This ratio reduces to the normalized Stoke's parameters $\frac{Q}{I}$ and $\frac{U}{I}$ for $\alpha=0^{\circ}$ and $\alpha=22.5^{\circ}$. In practice, additional measurements are made at two more values of $\alpha$, namely $45^{\circ}$ and $67.5^{\circ}$, for reasons explained in Sect. 5.

The half-wave plate and the Wollaston prism are placed in between a field lens-camera lens combination, which reimages the telescope focal plane on to the main CCD with a reduction factor of about 3.8; the field lens reimages the telescope aperture on the half-wave plate and the light reaches the camera lens without any vignetting. As shown in Fig. 1 each point in the telescope focal plane produces two images on the CCD, corresponding to the ordinary and the extraordinary beams. In order to avoid overlap of the images of adjacent points, for observations of extended objects, a grid of parallel obscuring strips is placed at the focal plane of the telescope. The width of and spacing between the strips are chosen in such a way as to avoid overlap of the ordinary and the extraordinary images on the CCD. Four $0.3 \mathrm{~mm}$ diameter holes are provided at the four corners of the grid and are used to focus the surface of the grid, which coincides with the focal plane of the telescope, onto the surface of the CCD. The grid is made of black dielectric material to avoid polarization of the stray light arising due to reflections from its edges. Besides, the edges are made slanted (Fig. 1) to prevent vignetting of the telescope beam.

The A\&G unit has a field of view of about $2^{\prime}$ and can be positioned anywhere within a $40 \times 100 \mathrm{~mm}^{2}$ area at the focal plane of the telescope close to the main field. This area has been chosen on the basis of the requirement that three stars brighter than $m_{v}=15$ should be available with $95 \%$ confidence level towards the poles of the Galaxy.

\subsection{Instrument control and data-acquisition}

A block schematic of the IMPOL control system is shown in Fig. 2. The electronics assembly of the instrument consists of three parts - (i) the positioning systems for the half-wave plate and the A\&G unit probe, (ii) the CCD camera for the A\&G unit and the associated electronics and (iii) the main CCD camera with its own control electronics and host computer (PC 486) for exposure control and data-acquisition. The important parameters of this CCD camera, which was also developed at IUCAA, are listed in Table 1 (for more details refer to Deshpande \& Gadre 1994).

\section{Sources of errors}

In addition to the fundamental limit due to photon statistics, the measurement of polarization is affected by several factors some of which are discussed in the following subsections. However, the effects of flat-field errors and their remedy needs particular attention and is treated in detail in Sect. 5. 


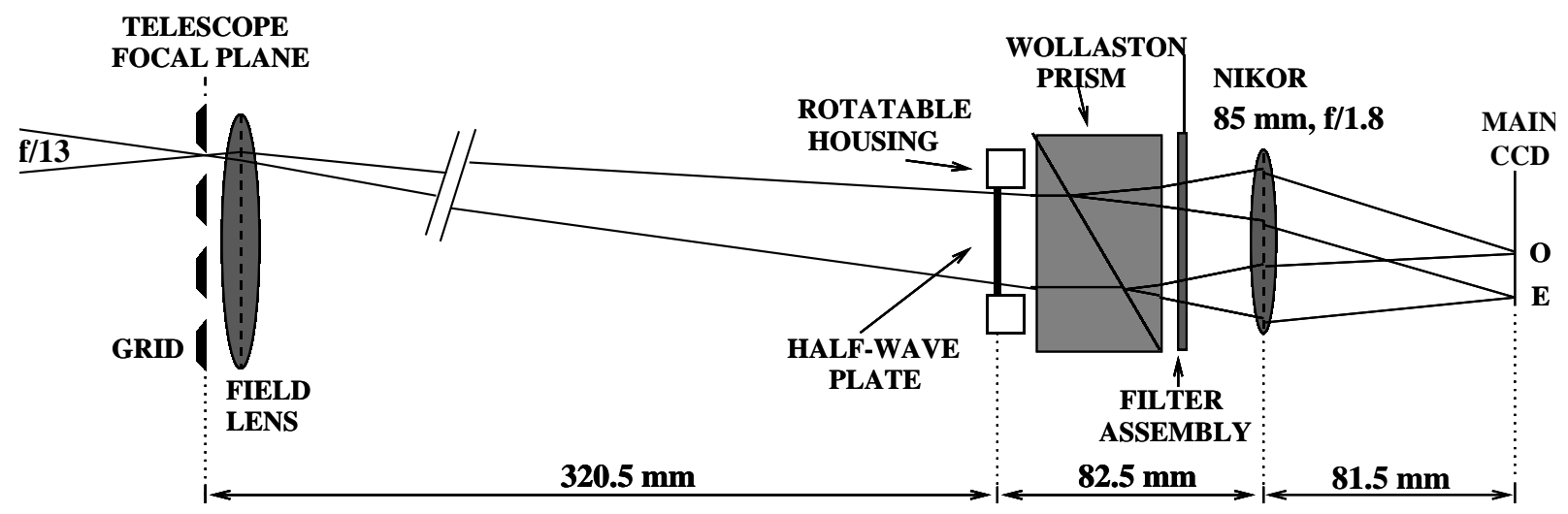

Fig. 1. Schematic of the IMPOL optical layout. The field lens and half-wave plate are from Karl Lambrecht (part Nos. 322305 and WPAC 2-22-BB400/700 respectively). The Wollaston prism is from Bernard Halle (PWQ 30.30) and the camera lens is Nikkor AF Telephoto $85 \mathrm{~mm}, \mathrm{f} / 1.8$

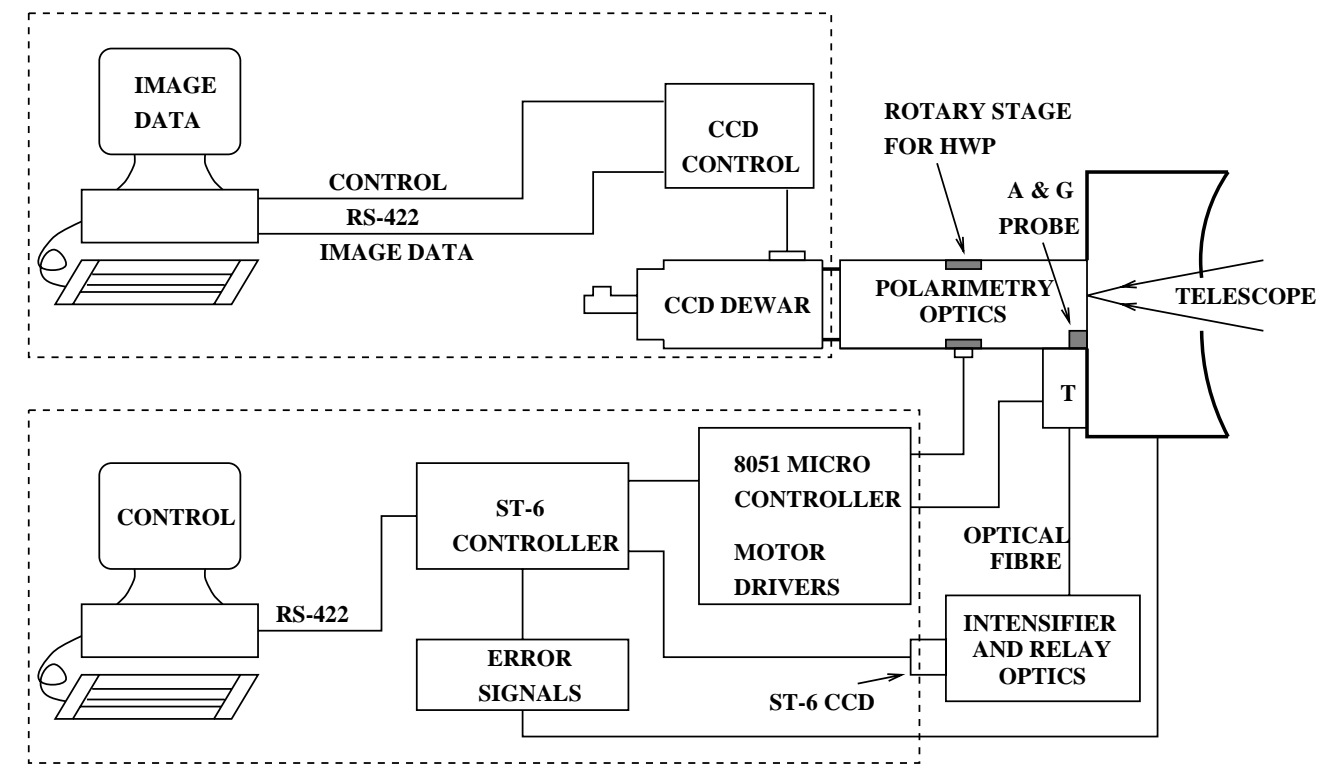

Fig. 2. Various blocks of the IMPOL control system. The block marked "T" represents the $X Y$ stages of the A\&G unit

\subsection{Atmospheric effects}

In IMPOL, two orthogonal polarization components are measured simultaneously and the Stoke's parameter is obtained from the ratio of the fluxes in these components (Eq. 2). Since the atmosphere is not birefringent, this eliminates the effects due to atmospheric scintillation, or for that matter any effect which changes both the polarization components by the same factor - like variations in the effective exposure times of observations, presence of thin clouds etc.

\subsection{Distortions in polarization}

All the optical elements are anti-reflection coated to minimize the polarization efffects due to reflection at their
Table 1. The parameters of IUCAA CCD camera

\begin{tabular}{ll}
\hline Parameters & Value \\
\hline CCD make & EEV CCD02-06 series \\
CCD chip size & $385(\mathrm{H}) \times 578(\mathrm{~V})$ \\
Pixel size & $22 \times 22 \mu \mathrm{m}$ \\
Active area & $12.7 \mathrm{~mm} \times 8.5 \mathrm{~mm}$ \\
No. of amplifiers & 1 \\
Quantum efficiency & blue: $20 \%$ \\
& yellow: $50 \%$ \\
Readout speed & red: $60 \%$ \\
Acquisition \& Display time & $32 \mu \mathrm{s}$ per pixel \\
Read noise (total) & $8 \mathrm{e}^{-}$rms full frame \\
Gain & $5 \mathrm{e}^{-} / \mathrm{ADU}$ \\
\hline
\end{tabular}


surfaces, and care is taken to minimize the stray light, reflected from the walls etc., reaching the detector. In particular, all the aperture stops in the beam path are chosen to be non-metallic, including the grid in the focal plane, so as to avoid polarization of light scattered from these (Pospergelis 1965). The mounts for field lens, the halfwave plate and the Wollaston prism have been designed to minimize stress-birefringence due to differential thermal expansion.

The retardance introduced by the half-wave plate could deviate from $180^{\circ}$ either because of the finite angle of incidence or because of the chromatic effects. If the beam is incident at a small angle $i$, the maximum change in retardance is given by (derived from expression in Serkowski 1975)

$\sigma_{\tau} \simeq \pi \times \frac{i^{2}}{4 n_{\mathrm{o}}}\left(\frac{1}{n_{\mathrm{o}}}-\frac{1}{n_{\mathrm{e}}}\right)$

where $n_{\mathrm{o}}$ and $n_{\mathrm{e}}$ are the refractive indices of the material for ordinary and extraordinary rays. For the aperture used, the maximum angle of incidence is about $5^{\circ}$ so that $\sigma_{\tau} \lesssim 0.01 \mathrm{rad}$. The chromatic effects give $\sigma_{\tau}<0.18 \mathrm{rad}$ for the wideband and $\sigma_{\tau}<0.14 \mathrm{rad}$ for the $V$-band. It can be shown (Serkowski 1974) that the depolarization $\sigma_{\mathrm{p}}$ due to an uncertainty of $\sigma_{\tau} \mathrm{rad}$ in the retardance is to the lowest order given by $\sigma_{\mathrm{p}} \simeq p \sigma_{\tau}^{2}$, $p$ being the fractional polarization. Therefore even for the wideband $\sigma_{\mathrm{p}} \lesssim 0.03 p$. Further, circular polarization in the incident light is converted to a linear polarization of magnitude $V \sigma_{\tau}$, where $V$ is the circularity parameter. But in typical observations this does not pose a serious problem because the circular polarization is usually less than the linear polarization.

If the position angle of the half-wave plate fast axis changes with wavelength, it renders the measurement of $\theta$ erroneous. The achromatic half-wave plate used in IMPOL does not produce any appreciable dispersion in its fast-axis position angle over the wavelength range of interest.

The uncertainty $\sigma_{\alpha}$ involved in the positioning of the half-wave plate leads to a maximum error in the measurement of linear polarization given by $\sigma_{\mathrm{p}} \simeq p \sigma_{\alpha}$. Thus, $\sigma_{\mathrm{p}} \simeq 0.002 p$ for $\sigma_{\alpha}=0.1^{\circ}$.

\subsection{Photon noise}

From Eq. (3) it can be shown that the variance due to photon noise in the measurement of each of the Stoke's parameters $R(\alpha)$ is given by

$\sigma_{R}^{2}=\frac{4 N_{\mathrm{o}} N_{\mathrm{e}}}{\left(N_{\mathrm{o}}+N_{\mathrm{e}}\right)^{3}}(1+k)$

where $N_{\mathrm{o}}$ and $N_{\mathrm{e}}$ are the number of photoelectrons and $k$ is the ratio of the flux from the background to that from the source. For small values of $p, N_{\mathrm{o}}$ and $N_{\mathrm{e}}$ are approx- imately equal and denoting $N=N_{\mathrm{o}}+N_{\mathrm{e}}$, the standard deviation of $p$ can be written as

$\sigma_{\mathrm{p}} \simeq 100 \times \frac{\sqrt{N+N_{B}}}{N} \%$,

where $N_{B}=k N$ for observations with the grid and $N_{\mathrm{B}}=2 k N$ for observations without the grid (see Sect. $5)$. Similarly, we can also show that

$\sigma_{\theta} \simeq 0.5 \times \frac{\sigma_{\mathrm{p}}}{p} \mathrm{rad}$

It is worth noting here that $p$, as defined above, is a positive definite quantity and follows the Rice probability distribution given by

$F\left(p, p_{0}\right)=\frac{p}{\sigma_{\mathrm{p}}} \mathrm{e}^{\frac{p^{2}+p_{0}^{2}}{2 \sigma_{\mathrm{p}}^{2}}} I_{0}\left(\frac{p p_{0}}{\sigma_{\mathrm{p}}^{2}}\right)$.

Here, $p_{0}$ is the true value of fractional polarization being estimated by $p$ and $I_{0}$ is the modified Bessel function of order zero. Since $p$ is a biased estimator, several schemes have been suggested (Simmons \& Stewart 1984 and references therein) for debiasing, but none of them is fully satisfactory. For values of $\frac{p}{\sigma_{\mathrm{p}}}$ larger than about 4 , almost all the debiasing schemes agree and reduce approximately to the relation

$\hat{p}_{0}=p \sqrt{1-\frac{\sigma_{\mathrm{p}}^{2}}{p^{2}}}$,

where $\hat{p}_{0}$ is the "debiased" estimate of $p_{0}$. Thus it is desirable to work with the normalized Stoke's parameters as far as possible and use the quantities $p$ and $\theta$ only to present the final results. However, in general, the normalized Stoke's parameters themselves might not be normally distributed and might have bias (Clarke et al. 1983). Besides, for photon-noise dominated measurements, the positive kurtosis of the distribution will lead to erroneous estimates of the confidence levels unless more than a few thousand photoelectrons are collected. Thus, in order to arrive at an optimum procedure, it is essential to carefully study the nature of the dominant sources of noise in the measurement and their effects on the analysis (see Sect. 5).

\section{Performance estimates}

In this section, we calculate the accuracy of polarization measurement due to photon noise for two typical types of observations. It is assumed that the observations are made in the $V$-band $\left(V_{0}=10^{6}\right.$ photons $\left.\mathrm{cm}^{-2} \mathrm{~s}^{-1}\right)$ using a telescope of effective area $\sim 10^{4} \mathrm{~cm}^{2}$, the brightness of the sky is 20 mag per sq arcsec, and that the software aperture used for photometry is about $30 \mathrm{sq}$ arcsec. The combined effect of the atmosphere, the telescope, the optics and quantum efficiency of the detector etc., is taken to result in an effective transmission of $\eta \sim 3.2 \%$. 
A classical example of polarimetric observations involves study of dark molecular clouds with the light of stars shining behind their periphery (e.g. Elvius 1970; Joshi et al. 1985; Kane et al. 1995). Typically the polarization of these sources is in the range $1-5 \%$. Assuming stars of apparent visual magnitude $m_{v}=15$, we see that the total number of photo-electrons collected, due to the star $(N)$ and the background $\left(N_{B}\right)$, within the software aperture, for an exposure of 15 minutes are $2.910^{5}$ and $8.610^{4}$ respectively. Therefore, the corresponding error in polarization measurement is given by

$\sigma_{\mathrm{p}}=100 \frac{\sqrt{2.910^{5}+8.610^{4}}}{2.910^{5}} \simeq 0.21 \%$.

If the observations are made with a filter which covers both the $V$ and $R$ bands $(\Delta \lambda \sim 200 \mathrm{~nm})$, there will be an improvement in the signal-to-noise ratio by a factor of $\sqrt{2}$, giving $\sigma_{\mathrm{p}} \sim 0.15 \%$.

Study of extended objects like reflection nebulae, is another field in which polarimetric observations are useful. Polarization in the light scattered from these clouds can range from a few percent to as much $30 \%$. Assuming that the observation involves a reflection nebula of the same surface brightness as the background sky, the integrated photoelectron count from each, for an exposure of 15 minutes, will be $8.610^{4}$ and hence

$\sigma_{\mathrm{p}}=100 \frac{\sqrt{8.610^{4}+8.610^{4}}}{8.610^{4}} \simeq 0.48 \%$.

for a solid angle defined by the software aperture. The wideband observations, in this case will give an error of about $0.34 \%$. In Fig. 3 the solid and dashed curves show the estimated error in the measurement of polarization, due to photon statistics alone, for the two cases discussed above, namely stellar sources and reflection nebulae respectively. The crosses indicate how well actual measurements conform to the errors predicted by photon noise alone.

\section{Observations and data-analysis}

We have seen in Sect. 2.1 that observations for at least two positions of the half-wave plate are required to determine the three unknown parameters namely, the total, intensity $(I)$, fraction of light in linearly polarized condition $(p)$, and position angle of the plane of polarization $(\theta)$. However, the situation is not so simple in reality because - (i) the responsivity of the system to the two orthogonal polarization components may not be the same, and (ii) the responsivity of the CCD is a function of the position on its surface. Due to these effects the signals which are actually measured in the two images $\left(I_{\mathrm{e}}^{\prime}\right.$ and $I_{\mathrm{o}}^{\prime}$ ) are given by

$I_{\mathrm{e}}^{\prime}(\alpha)=I_{\mathrm{e}}(\alpha) F_{\mathrm{e}} \quad$ and

$I_{\mathrm{o}}^{\prime}(\alpha)=I_{\mathrm{o}}(\alpha) F_{\mathrm{o}}$,

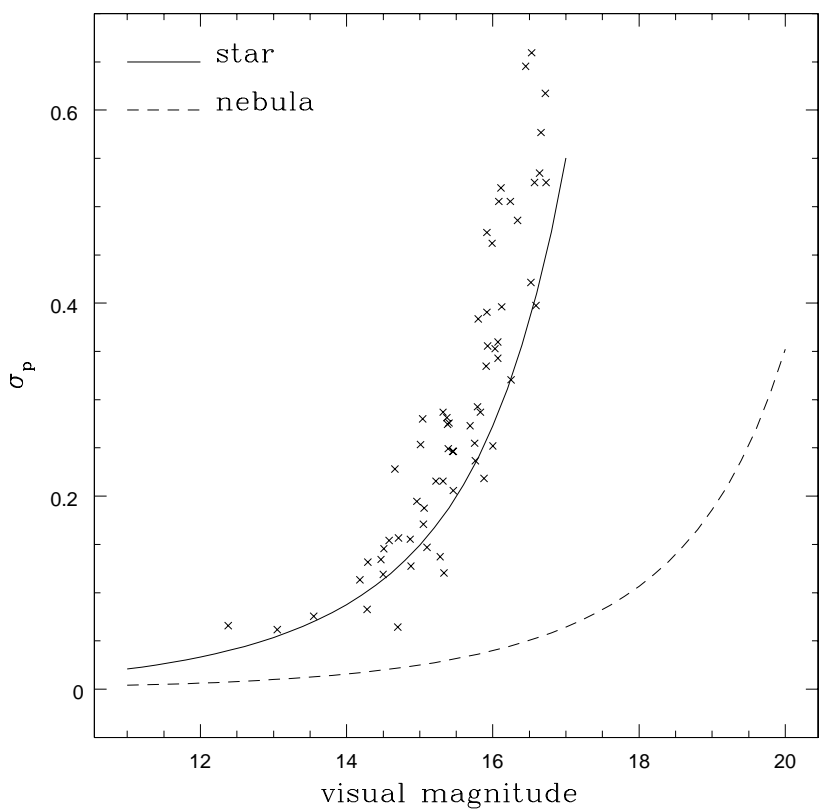

Fig. 3. The errors due to photon noise alone, as calculated in Sect. 4, for wideband measurements of fractional polarization $p$ are shown as a function of the brightness of the source. The solid curve refers to the case of stars while the dashed curve is for an extended object, for which the $x$-axis represents the surface brightness of the source in magnitudes per sq arcsecs. The background is assumed to be $20^{\text {th }}$ magnitude per sq arcsec and a software aperture of $30 \mathrm{sq}$ arcsec has been used during data reduction. The crosses represent the errors in actual polarimetric measurements made with instrument on a field at the periphery of the dark cloud B133

where $F_{\mathrm{e}}$ and $F_{\mathrm{o}}$ represent the effects mentioned above. In order to ensure that $F_{\mathrm{e}}$ and $F_{\mathrm{o}}$ do not change during observations of an object, the positions of the two images are kept fixed on the CCD. Further, as the analyzer is fixed with respect to the detector, the orientation of the ordinary and extraordinary polarizations also remain fixed with respect to it, independent of the polarization vector of the incident beam. Therefore the ratio of the factors $F_{\mathrm{o}}$ and $F_{\mathrm{e}}$ can be estimated as

$\frac{F_{\mathrm{o}}}{F_{\mathrm{e}}}=\left[\frac{I_{\mathrm{o}}^{\prime}\left(0^{\circ}\right)}{I_{\mathrm{e}}^{\prime}\left(45^{\circ}\right)} \times \frac{I_{\mathrm{o}}^{\prime}\left(45^{\circ}\right)}{I_{\mathrm{e}}^{\prime}\left(0^{\circ}\right)} \times \frac{I_{\mathrm{o}}^{\prime}\left(22.5^{\circ}\right)}{I_{\mathrm{e}}^{\prime}\left(67.5^{\circ}\right)} \times \frac{I_{\mathrm{o}}^{\prime}\left(67.5^{\circ}\right)}{I_{\mathrm{e}}^{\prime}\left(22.5^{\circ}\right)}\right]^{\frac{1}{4}}$,

by making use of the fact that a rotation of the half-wave plate by $45^{\circ}$ simply leads to an interchange of the signals in the ordinary and extraordinary images. Now the actual ratio of the fluxes in the two images may be recovered as

$\frac{I_{\mathrm{e}}(\alpha)}{I_{\mathrm{o}}(\alpha)}=\frac{F_{\mathrm{o}}}{F_{\mathrm{e}}} \times \frac{I_{\mathrm{e}}^{\prime}(\alpha)}{I_{\mathrm{o}}^{\prime}(\alpha)}$.

This ratio is substituted in Eq. (2) and a cosine curve is fitted to the four values of $R(\alpha)$ obtained so as to make the best estimates of $p$ and $\theta$. 
Since CCDs exhibit both intrapixel as well as pixel to pixel sensitivity variations, ideally the images should be stable to within a small fraction of a pixel during exposures at different positions of the half-wave plate. But since this is difficult to achieve, it is advisable to dither the telescope pointing atleast by \pm 0.5 pixels along each axis and to get an FWHM of greater than 3 pixels.

Since the grid placed at the telescope focal plane covers almost half the field, several exposures at slightly different telescope orientations might be required to cover the entire object field. Alternatively, in the case of stellar fields with slowly changing (in intensity and polarization) background, the grid may be removed during observations, provided the field is not too crowded.

A polarimetry package has been developed for data analysis within the IRAF environment using a mixture of standard IRAF tasks, custom-made CL scripts and FORTRAN routines. PSF fitting tasks of the DAOPHOT package are used to determine accurately the centroids of the stellar images. The intensity estimates are, however made using aperture photometry covering a diameter greater than $2 \mathrm{FWHM}$ so as to integrate more than $90 \%$ of the signal.

In order to keep a check on the errors, it is useful to take multiple exposures for each position of the halfwave plate. To ensure that the normalized Stoke's parameters follow a normal distribution as closely as possible, the signals measured at each position of the half-wave plate, should be averaged together before taking their ratios to give the normalized Stoke's parameters. However, this method has its demerits too. Firstly, it leaves the nonlinear $\chi^{2}$ fitting technique with only one degree of freedom, to estimate the two parameters $p$ and $\theta$ from the four values of $R(\alpha)$. Secondly, it does not allow the estimation of errors in the measurement of the Stoke's parameters, since there are not enough degrees of freedom. Therefore, it appears optimum to use a fitting technique which estimates $p$ and $\theta$, from all the values of $R(\alpha)$ obtained from individual exposures.

\section{Commissioning of IMPOL}

The first phase of commissioning involved laboratory tests to determine various parameters like linearity, gain and readnoise of the detector, vignetting in the optics etc. Measurements were also made with a $3000 \mathrm{~K}$ filament source to estimate depolarization and instrumental polarization in $B, V, R$ bands, and a wide band defined by a $3 \mathrm{~mm}$ thick KG3 glass. A $100 \%$ polarized beam showed that the depolarization is about $0.4 \%$ in the $B$ and $V$ bands, less than $1 \%$ in the $R$ band, and less than $6 \%$ in the broad band. There was no discernible rotation of the position angle between these bands. For $R$ and the wideband, the accuracy of measurements is limited by the performance of the polarizer used. Measurements made with a Lyot depolarizer introduced in front of the artificial star showed that the instrumental polarization is about $0.03 \%$ in the $B$-band and less than $0.06 \%$ with the wideband filter.

The second stage of tests were conducted when the instrument saw the first light in February 1996, at the $\varnothing 1.2 \mathrm{~m}$ Gurushikhar Infra-Red Telescope facility on Mt. Abu, Rajasthan, operated by the Physical Research Laboratory at Ahmedabad, India. Table 2 contains the results of some of the observations of standard stars in the $V$-band. Column 1 of the table gives the identification number of the star. Columns 2-5 contain the measured values and $1 \sigma$ errors of the fractional polarization $p$ and the position angle $\theta$. Column 6 gives the error $\sigma_{\mathrm{p}}$ based on photon statistics alone, derived as illustrated in Sect. 3 . Columns 7 and 8 list the published values of $p$ and $\theta$.

The first two stars in the table are polarized standards while the last two are unpolarized ones. The four measurements of star HD 39587 are made at different corners of the CCD frame in order to verify the uniformity of response. Comparing the measured and published results for the polarized stars we see that there is no indication of depolarization.

Table 2. Observations of standard stars

\begin{tabular}{|c|c|c|c|c|c|c|c|}
\hline $\begin{array}{c}\text { Star } \\
(1)\end{array}$ & $\begin{array}{c}p \\
(2)\end{array}$ & $\begin{array}{l}\sigma_{\mathrm{p}} \\
(3)\end{array}$ & $\begin{array}{c}\theta \\
(4)\end{array}$ & $\begin{array}{l}\sigma_{\theta} \\
(5)\end{array}$ & $\begin{array}{c}\sigma_{\text {phot }} \\
(6)\end{array}$ & $\begin{array}{l}p_{0} \\
(7)\end{array}$ & $\begin{array}{l}\theta_{0} \\
(8)\end{array}$ \\
\hline HD & $\%$ & $\%$ & $\circ$ & $\circ$ & $\%$ & $\%$ & $\circ$ \\
\hline 43384 & 3.05 & 0.04 & 172 & 0.3 & 0.03 & 3.0 & 170 \\
\hline 154445 & 3.65 & 0.03 & 90.5 & 0.2 & 0.02 & 3.7 & 90 \\
\hline 102870 & 0.03 & 0.01 & 64.4 & 7.8 & 0.02 & 0.017 & 162 \\
\hline \multirow[t]{4}{*}{39587} & 0.03 & 0.03 & 140 & 27.5 & 0.02 & \multirow[t]{4}{*}{0.013} & \multirow[t]{4}{*}{20} \\
\hline & 0.05 & 0.02 & 159 & 12.7 & 0.02 & & \\
\hline & 0.09 & 0.02 & 34 & 6.7 & 0.02 & & \\
\hline & 0.05 & 0.02 & 167 & 11.3 & 0.02 & & \\
\hline
\end{tabular}

Neither is there any rotation of the position angle beyond a couple of degrees. From Col. 3 and Col. 6 for the unpolarized stars, we see that the error in the measured polarization is comparable with that expected on the basis of photon statistics alone.

In Fig. 4, the values of $q=R\left(0^{\circ}\right)$ are plotted against $u=R\left(22.5^{\circ}\right)$ for all the unpolarized standards observed in the $V$-band. The extremely low correlation coefficient between $q$ and $u$, is indicative of the low instrumental polarization. The average values of $q$ and $u$, give a value of polarization $p=0.03 \%$. These observations show that for accuracies $\widetilde{>} 0.05 \%$ in the measurement of polarization $p$, the performance is still limited by photon noise and the instrument polarization floor has not yet reached. 


\section{Summary}

An Imaging Polarimeter (IMPOL) has been constructed which has a field of view of about $6.5^{\prime}$ for a $\varnothing 1.2 \mathrm{~m}, \mathrm{f} / 13$ telescope. An off-axis acquisition and guidance unit capable of using stars as faint as $V_{\text {mag }} \sim 15$ is also built in the instrument so that long exposures of faint extended objects like reflection nebulae etc. can be taken while keeping the image fixed on the CCD face to within one half

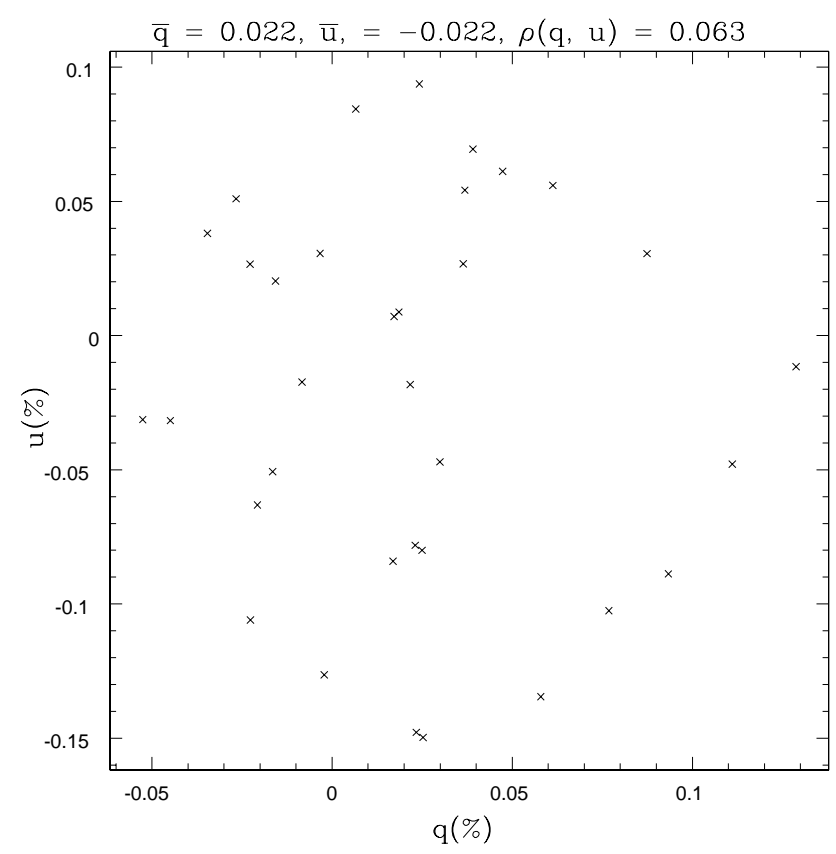

Fig. 4. Normalized Stoke's parameter $q$ is plotted against $u$ for a number of unpolarized standard stars. The correlation coefficient of the points is about 0.06 and the average values of $q$ and $u$ give a value of $p=0.03 \%$

of a pixel - this stability of the image is a necessary condition to achieve a high accuracy in relative photometry between the different frames used to estimate the polarization. Observations of nearby standard polarized and unpolarized stars show that for wideband observations, there is no discernible depolarization and the instrumental polarization is less than $0.05 \%$. Preliminary results of wideband polarimetry of stellar fields, using a PSF fitting technique for determining the centroids of the stellar images and aperture photometry to derive the intensities of the individual images, give close to photon-noise limited accuracy of $0.15 \%$ for $V_{\text {mag }}=15$ stars with about an hour of total exposure time. For extended objects with brightness about 20th mag per square arcseconds and a background of about the same brightness, it is estimated that an accuracy of about $1 \%$ is possible with 60 minutes of total exposure time and an aperture of $10 \mathrm{sq}$ arcsec.

Acknowledgements. The project has been funded by the Department of Science and Technology of the Government of India. The authors also wish to thank the Physical Research Laboratory, Ahmedabad for providing telescope time, P.A. Chordia and R. Bedade for their involvement in developing the electronic circuitry for the instrument and M.S. Deshpande for her assistance during its commissioning. Thanks are also due to Dr. A. Saha and an anonymous referee for their valuable comments.

\section{References}

Appenzeller I., 1967, PASP, 136

Clarke D., Stewart B.G., Schwarz H.E., Brooks A., 1983, A\&A 126,260

Deshpande M.S., Gadre D.V., 1994, in: Proceedings of the Indo-US Workshop on Array detectors and Image processing, Tandon S.N. and Ramaprakash A.N. (eds.) IUCAA, India, p. 237

Elvius A., 1970, in: Dark nebulae, Globules and Protostars, Lynds B.T. (ed.). The University of Arizona Press, Tucson, p. 57

Jannuzi B.T., Smith P.S., Elston R., 1993, ApJS 85, 265

Jarrett T.H., Novak G., Xie T., Goldsmith P.F., 1994, ApJ 430,743

Joshi U.C., Kulkarni P.V., Bhatt H.C., Kulshrestha A.K., Deshpande M.R., 1985, MNRAS 215, 275

Kane B.D., Clemens D.P., Leach R.W., Barvainis R., 1995, ApJ 445, 269

Kastner J.H., Weintraub D.A., 1994, ApJ 434, 719

Moore T.J.T., Yamashita, T., 1995, ApJ 440, 722

Ohman Y., 1939, MNRAS 99, 624

Pospergelis M.M., 1965, SvA-AJ 9, 313

Scarrott S.M., Warren-Smith R.F., Pallister W.S., Axon D.J., Bingham R.G., 1983, MNRAS 204, 1163

Scarrott S.M., 1991, Vist. Astron. 34, 163

Sen A.K., Tandon S.N., 1994, in: Instrumentation in Astronomy VIII, Crawford D.L. (ed.), SPIE proceedings, Vol. 2198, part 1, p. 264

Serkowski K., 1974, in: Methods of Experimental Physics: Astrophysics, Carleton N. (eds.) Vol. 12, part A Academic Press, p. 361

Serkowski K., 1975, in: Planets, Stars and Nebulae studied with photopolarimetry, Gehrels T. (ed.). The University of Arizona Press, Tucson, p. 135

Simmons J.F.L., Willis J.P., Newsam A.M., 1995, A\&A 293, L46

Simmons J.F.L., Stewart B.G., 1984, A\&A 142, 100

Weintraub D.A., Kastner J.H., Whitney B.A., 1995, ApJ 452, L141

Wolstencroft R.D., Done C.J., Scarrott S.M., Scarrott R.M.J., 1995, MNRAS 276, 460 